\title{
Unravelling Competing Oxidation Mechanisms in Single Cu Nanoparticles
}

\section{Sara Nilsson}

Chalmers University of Technology

\section{Monia Nielsen}

Technical University of Denmark

Joachim Fritzsche

Chalmers Univerity of Technology https://orcid.org/0000-0001-8660-2624

\section{Christoph Langhammer}

Chalmers University of Technology https://orcid.org/0000-0003-2180-1379

Shima Kadkhodazadeh ( $\sim$ shka@dtu.dk)

Technical University of Denmark https://orcid.org/0000-0003-3606-8851

\section{Article}

Keywords:

Posted Date: January 20th, 2022

DOI: https://doi.org/10.21203/rs.3.rs-1148708/v1

License: (c) (1) This work is licensed under a Creative Commons Attribution 4.0 International License. Read Full License 


\section{Unravelling Competing Oxidation}

\section{Mechanisms in Single Cu Nanoparticles}

Sara Nilsson ${ }^{1, \dot{\dagger}}$, Monia Runge Nielsen ${ }^{2}$, Joachim Fritzsche ${ }^{1}$, Christoph Langhammer ${ }^{*, 1}$ and Shima Kadkhodazadeh $* *, 2, \dagger$

${ }^{1}$ Department of Physics, Chalmers University of Technology, 41296 Göteborg, Sweden

${ }^{2}$ DTU Nanolab, Technical University of Denmark, Fysikvej, 2800 Kgs Lyngby, Denmark

$\dagger$ 'equal contribution

*clangham@chalmers.se;**shka@dtu.dk 


\begin{abstract}
Chemical reactions involving nanoparticles often follow complex processes. In this respect, real-time probing of single particles under reactive conditions is crucial for uncovering the mechanisms driving the reaction pathway. Here, we have captured in situ the oxidation of single $\mathrm{Cu}$ nanoparticles to unravel a sequential competitive activation of different mechanisms at temperatures $50-200{ }^{\circ} \mathrm{C}$. Using environmental scanning transmission electron microscopy, we monitor the evolution of oxide formation with sub-nanometer spatial resolution and show how different mechanisms closely determine the morphology of the particles. Moreover, using in situ electron energy-loss spectroscopy, we probe the localized surface plasmons of individual particles during oxidation, and with the aid of finite-difference time-domain electrodynamic simulations, investigate the signature of each mechanism in their plasmonic response. Our results shed light on the intricate processes involved in the oxidation of metallic nanoparticles, valuable for applications in catalysis, electronics, nanomedicine and plasmonics.
\end{abstract}




\section{Introduction}

Understanding oxidation processes and the corresponding mechanisms involved is paramount for the design, synthesis and fabrication of technologically relevant metal nanostructures ${ }^{1,2}$. Focusing on $\mathrm{Cu}$, its nanoparticles both in metallic and oxide forms are attractive for applications within electronics ${ }^{3,4}$, photonics ${ }^{5,6}$, catalysis ${ }^{7,8}$ and nanomedicine ${ }^{8}$, owing to $\mathrm{Cu}^{\text {'s }}$ unique physical and chemical properties, and relatively high abundance. However, applications of metallic $\mathrm{Cu}$ particles are limited due to their chemical instability at ambient conditions. At the same time, oxide-covered $\mathrm{Cu}$ nanoparticles are highly interesting for catalysis ${ }^{9,10}$ and active plasmonics ${ }^{5,11,12}$. Moreover, hollow oxide nanoparticles synthesized by exploiting the nanoscale Kirkendall (NK) effect $^{13,14}$ can be advantageous in sensor technologies ${ }^{15,16}$ and in nanomedicine ${ }^{8}$, due to their geometry and high surface area. Since the seminal report of the NK effect in Co nanoparticles ${ }^{17}$, other metals including $\mathrm{Cu}^{18}, \mathrm{Ni}^{19}$ and $\mathrm{Fe}^{20}$, have been shown to undergo the same transformation to hollow structures upon oxidation. This effect, caused essentially by the higher diffusion rate of cationic metal ions through the oxide compared to oxygen ions, is manifested initially as the nucleation of small vacancies, which then accumulate to form nanoscale voids ${ }^{18,21}$. Other oxidation mechanisms, such as the Cabrera-Mott ${ }^{22}$ and Valensi-Carter ${ }^{23}$ processes, have also been reported for nanoparticles and thin films ${ }^{24-26}$, as well as the Johnson-Mehl-Avrami-Kolmogorov (JMAK) nucleation of oxide islands ${ }^{27,28}$ at the initial stages of thin film oxidation. Overall, a holistic and detailed understanding of the entire oxidation pathway of metal nanoparticles, and in particular of $\mathrm{Cu}$ nanoparticles, is, however, still lacking. In part, this is because the majority of the existing in situ studies are based on particle ensembles ${ }^{29-31}$ and thus, inherently suffer from averaging effects. The few existing in situ single particle studies using scanning transmission electron microscopy $(\mathrm{STEM})^{32}$ and plasmonic nanospectroscopy ${ }^{33}$ are limited in that the former was executed at very low oxygen pressures, and the latter is blind to morphological details. Here, we address these challenges by recording in situ the morphological and compositional evolution of single $\mathrm{Cu}$ nanoparticles using annular dark-field STEM (ADF STEM) imaging, and by simultaneously probing the evolution of their localized surface plasmon resonances (LSPR) using electron energy-

loss spectroscopy (EELS) ${ }^{6,34-36}$ in an environmental TEM (ETEM) ${ }^{37}$ (Fig. S1), in analogy to the bulk plasmon studies of hydride formation in Pd nanocrystals ${ }^{38}$. Based on our direct observations, we are able to identify the active oxidation mechanisms involved, and thus, uncover the subsequent oxidation pathways at different temperatures. Moreover, by combining our EELS results with finite-difference time-domain (FDTD) electrodynamic simulations, we determine the signature of different oxidation mechanisms in the LSPR of the particles.

\section{In situ ADF STEM of single Cu nanoparticle oxidation at elevated temperatures}

Polycrystalline $\mathrm{Cu}$ nanodisks with radius $R=43 \pm 7 \mathrm{~nm}$ and nominal $20 \mathrm{~nm}$ thickness were fabricated by hole-mask colloidal lithography ${ }^{39}$ on TEM substrates with integrated resistive heaters. First, samples were annealed inside the ETEM for 60 minutes at $400{ }^{\circ} \mathrm{C}$ in $3 \mathrm{mbar}_{2}$ to remove hydrocarbons and to recrystallize the disks into dome-shaped particles with few grains and 
$R=35 \pm 5.0 \mathrm{~nm}$. They were then oxidized in $3 \mathrm{mbar}^{\mathrm{O}_{2}}$ at $50-200{ }^{\circ} \mathrm{C}$. Selected ADF STEM images recorded during oxidation are depicted in Fig. 1a.

Starting at $50{ }^{\circ} \mathrm{C}$, we observe that oxide islands nucleate and grow on the particle surface (arrows in Fig. 1b, Fig. S2.1), consistent with previous observations for extended $\mathrm{Cu}$ surfaces at early stages of oxidation ${ }^{27,40}$. The oxide islands subsequently coalesce into a complete layer (identified as mostly $\mathrm{Cu}_{2} \mathrm{O}$ - see SI Fig. S2.4), which then grows homogeneously and reaches a self-limiting thickness of $5.5 \pm 0.7 \mathrm{~nm}$ after 90 min of accumulated $\mathrm{O}_{2}$ exposure. As a second key point, we observe a distinct gap of accumulated vacancies between the oxide shell and the metal core (Fig. 1c). At $100{ }^{\circ} \mathrm{C}$, the nanoparticles initially undergo a similar sequence of events, including the nucleation of oxide islands on the surface, and their coalescence into a continuous oxide layer separated from the metal core by a vacancy gap (Fig. 1a). However, at this temperature, the oxide shell does not self-passivate but enters a new oxidation stage, where a Kirkendall void nucleates at the metal - vacancy gap interface, and expands inwards into the metal core. During this expansion, the void initially keeps an approximately planar growth front towards the shrinking $\mathrm{Cu}$ core (Fig. 1d). Interestingly, after the void has expanded approximately half-way across the particle, the growth front reorients and begins expanding angularly into the remaining half of the metal core (images at 60 and $90 \mathrm{~min}$ at $100{ }^{\circ} \mathrm{C}$ in Fig. 1a). Raising the temperature to $150{ }^{\circ} \mathrm{C}$ increases the oxidation rate and although the nanoparticles undergo very similar processes to 100 ${ }^{\circ} \mathrm{C}$ (Fig. 1a), we also notice important differences. One is the coarser morphology of the oxide shell, characterized by larger grains and increased surface roughness (Fig. 1f). The other interesting difference is the formation of a thin inner oxide shell, separated from the outer oxide shell by a vacancy gap (Fig. 1e). At $200{ }^{\circ} \mathrm{C}$, the overall oxidation sequence and particle morphology is very similar to $150^{\circ} \mathrm{C}$ but the process occurs at an even higher rate. An important aspect to emphasize is the growth of a single void for the majority of $\mathrm{Cu}$ nanoparticles with $R_{0}<$ $40 \mathrm{~nm}$ oxidized at 100, 150 and $200{ }^{\circ} \mathrm{C}$ (Fig. S2.2). Alternative scenarios, such as the nucleation of multiple voids, are rare but become more frequent for larger and more polycrystalline particles (Fig. S2.3). 


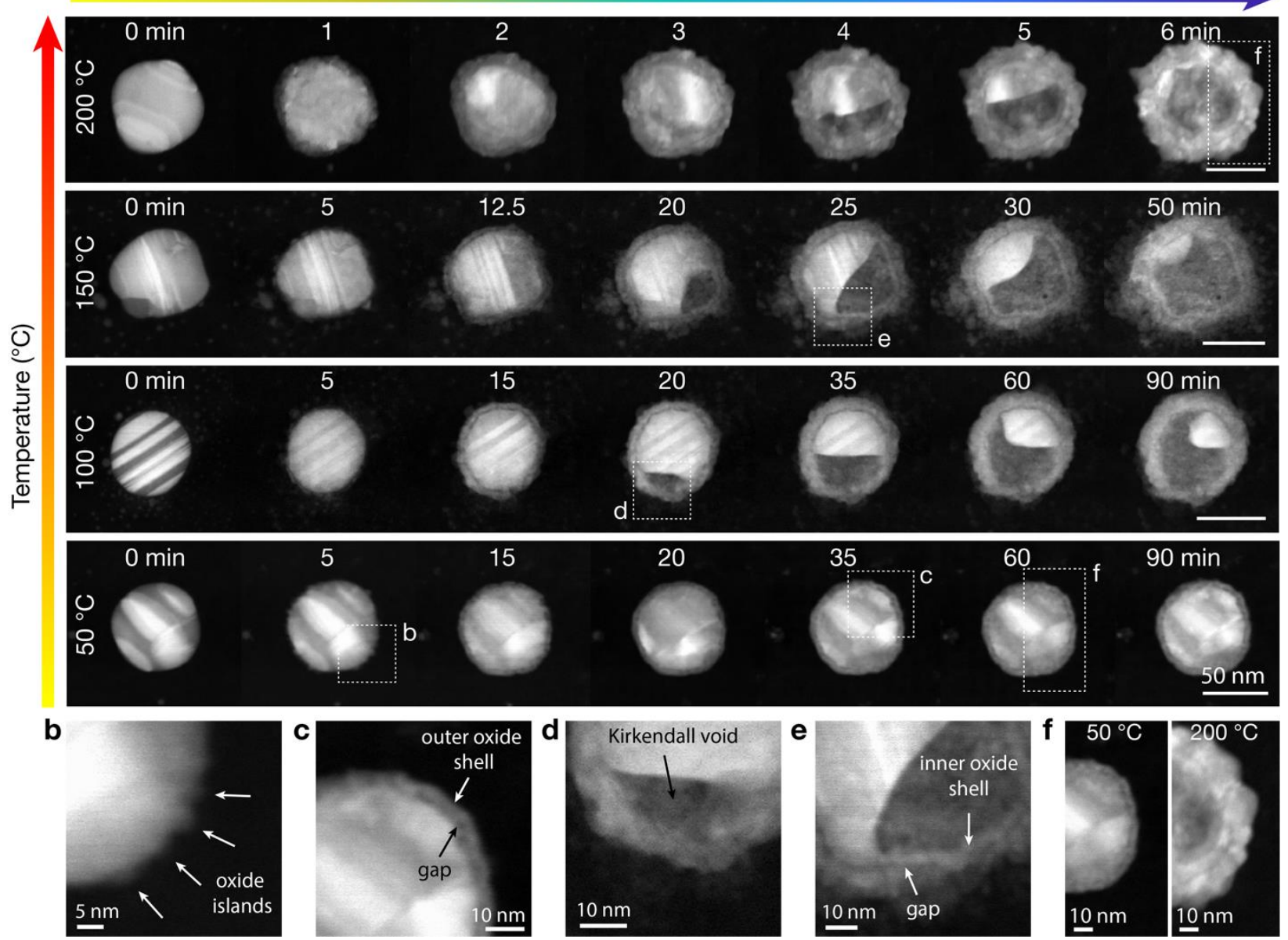

Figure 1. ADF STEM of single Cu particle oxidation at different temperatures. (a) ADF STEM images acquired during the oxidation of $\mathrm{Cu}$ nanoparticles at 50, 100, 150 and $200{ }^{\circ} \mathrm{C}$, in $3 \mathrm{mbar}$ $\mathrm{O}_{2}$, after different $\mathrm{O}_{2}$ exposure times (indicated in the images). Scale bars are $50 \mathrm{~nm}$. Specifically relevant features are highlighted: (b) the nucleation of oxide islands on the metallic surface at the initial stage of oxidation, (c) the formation of a homogeneous outer oxide shell after oxide island coalescence, and the vacancy gap layer between the outer shell and the metal core, (d) the typical NK void nucleation site at the metal - gap layer interface, (e) the inner oxide shell formed inside the particle and separated from the outer oxide shell by the vacancy gap layer, and (f) a comparison of the oxide morphology for oxidation at $50{ }^{\circ} \mathrm{C}$ (left) and $200{ }^{\circ} \mathrm{C}$ (right), revealing larger grain size and increased surface roughness at $200^{\circ} \mathrm{C}$.

\section{In situ EELS during oxidation at $50^{\circ} \mathrm{C}$}

To investigate the signature of the mechanistic evolution of $\mathrm{Cu}$ particles oxidized at $50{ }^{\circ} \mathrm{C}$ in their LSPR response, we employed in situ EELS (Fig. 2) in combination with FDTD simulations (Fig. 2d-e and SI Section S4.1-4.2) that explicitly take oxide island nucleation (cf. Fig. 1b), selflimiting oxide shell growth and vacancy gap formation stages (cf. Fig. 1c) into account. This way, we correlate the intricate morphological changes observed in the images with the LSPR from 
single nanoparticles. Figure 2 shows the time evolution of the LSPRs appearing in the EELS signal at two sets of perpendicular electron beam positions of a single $\mathrm{Cu}$ particle (ADF STEM images in Fig. 2a). At the general level, the two perpendicular LSPR modes follow very similar trends as the volume fraction of the metal converted to oxide, $\delta$, increases, due to the maintained symmetry of the particle during oxidation, and they are in reasonable agreement with the corresponding FDTD simulations (Fig. 2d-e). The smaller change in the relative intensity of the peaks in the simulations compared to EELS is likely because the simulations concern absorption extracted in the far-field, whereas EELS is a near-field probe ${ }^{41}$. Moreover, slight geometrical differences between the experiment and the model, e.g. defects formed during oxidation, are not included in the simulations ${ }^{42,43}$.

During the initial nucleation of oxide islands on the particle surface (at $\delta \approx 0-10 \%$ ), we observe either no change or a slight blue-shift of the LSPR in the EELS data. This is not reproduced in the simulations, which don't include changes in the surface electronic structure of $\mathrm{Cu}$ induced by oxygen adsorption and dissociation ${ }^{44}$. Since the LSPR of the $\mathrm{Cu}$ particles overlaps with the interband transition edge, we speculate that the observed blue-shift is a consequence of an increasing interband transition edge energy, due to the downward shift of the d-band center in the surface atoms chemically bonded to oxygen. This pushes the interband transition edge away from the LSPRs and reduces their damping. The initial stage is followed by a second regime ( $\delta=10-$ $15 \%$ ), characterized by rapid red-shift and intensity increase of the LSPR, which eventually slows down and ceases at $\delta \sim 20 \%$. These details are captured nicely by the FDTD model, and relate to the formation of the homogeneous oxide shell (dashed line in Fig. 2d-e) that induces spectral redshift and intensification upon its further growth. This red-shift halts when the self-limiting oxide thickness is reached, which occurs at $\delta \sim 20 \%$. These results are in good agreement with plasmonic nanospectroscopy of a similar system ${ }^{33}$ and signify how closely optical response and chemical and morphological properties of the particles are linked. For similar analysis of additional particles see SI Fig. S2.5. 
a
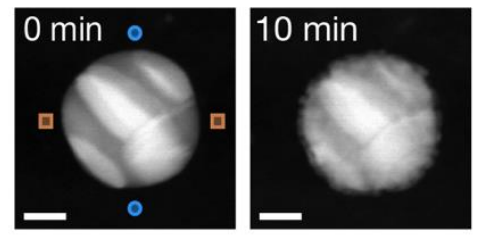

$90 \mathrm{~min}$

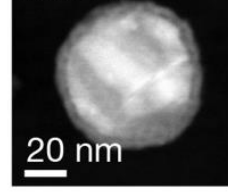

b

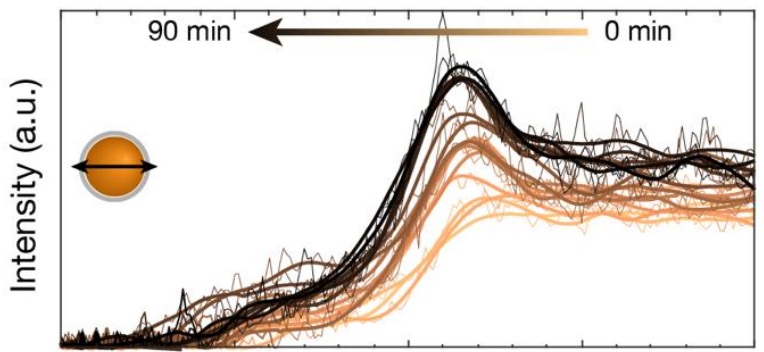

C

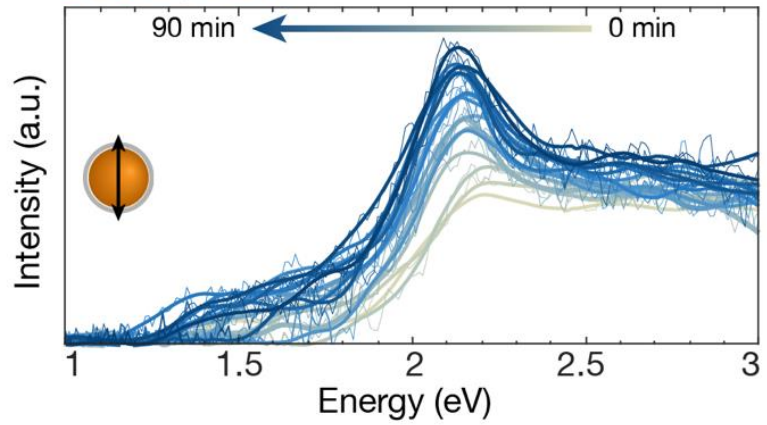

d

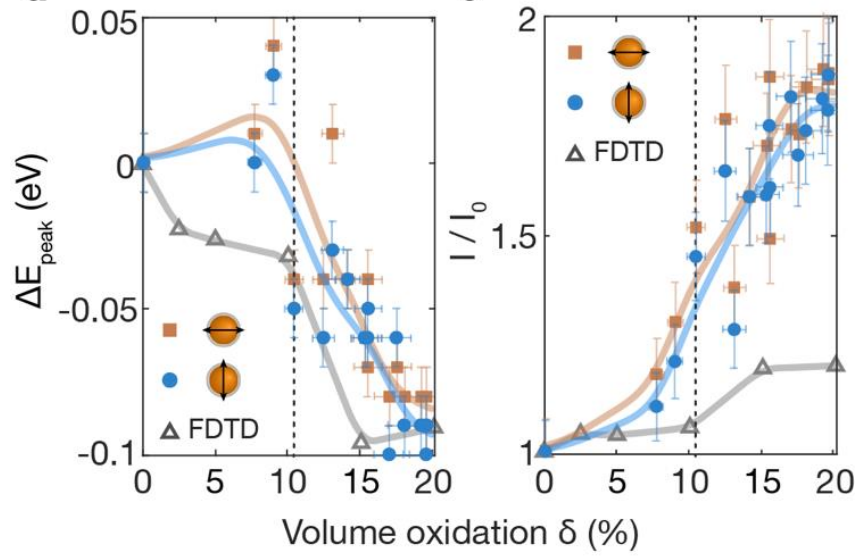

Figure 2. In situ EELS of single Cu nanoparticle oxidation at $50{ }^{\circ} \mathrm{C}$. (a) Selected ADF STEM images of a $\mathrm{Cu}$ nanoparticle oxidized at $50{ }^{\circ} \mathrm{C}$ (see Fig. 1 for additional images). (b, c) EELS signals from the particle recorded during oxidation with the electron beam positioned as specified in (a), revealing the LSPR signal of the particle. (d) The shift in the peak energy, $\Delta E_{\text {peak }}$, and (e) the relative peak intensity, $I / I_{0}$, of the $L S P R$ response as functions of volume oxidation fraction, $\delta$, (squares and circles) plotted together with the results of corresponding FDTD simulations for two perpendicular polarizations (triangles). The error bars in $\Delta E_{\text {peak }}, I / I_{0}$ and $\delta$ correspond to the energy dispersion per pixel in the EELS measurements, the average uncertainty of the fitted peak intensity, and the average uncertainty of the image segmentation process, respectively. The dashed lines indicate transition from oxide island to homogeneous oxide shell growth. 


\section{In situ EELS during oxidation at $100-200{ }^{\circ} \mathrm{C}$}

To map the evolution of the LSPR modes in single $\mathrm{Cu}$ nanoparticles oxidized at 100, 150 and $200{ }^{\circ} \mathrm{C}$, we performed identical in situ measurements to those described in the previous section. At these higher temperatures, beyond an initial oxide shell nucleation and growth, NK voids form and expand inside the particles. This is characterized by two subsequent phases of linear and angular void expansion, as introduced earlier (cf. Fig. 1 and Fig. S2.2). Analyzing the evolution of the EELS spectra recorded parallel and perpendicular to the linear NK void growth front reveals a more complex LSPR response compared to oxidation at $50{ }^{\circ} \mathrm{C}$ (Fig. 3b-c and Fig. S2.7). For $\delta$ $<20 \%$, the two LSPR modes behave similarly to the $50{ }^{\circ} \mathrm{C}$ case, and increase alike in intensity and red-shift. This is the consequence of the symmetric profile of the $\mathrm{Cu}$ core during this stage of oxidation. At $\delta \approx 20-25 \%$, this trend ends and instead the LSPR modes split into two peaks, accompanied by a distinct drop in intensity (Fig. 3d-e). The corresponding ADF STEM images (Fig. 3a and cf. Fig. 1) reveal that mode splitting signifies transition to the NK regime, upon which the circular symmetry of the $\mathrm{Cu}$ core breaks. Overall, the evolution of the LSPRs with oxidation is in good agreement with our FDTD simulations, and in situ optical plasmonic nanospectroscopy ${ }^{33}$. Small differences could stem from the thin inner oxide shell, which is not included in the FDTD model (Fig. 3d-e; details in SI Section S4.3). During the linear phase of void growth $(\sim 20 \%<\delta<\sim 60 \%)$, the two modes diverge, as the aspect ratio of the remaining metallic core increases, resulting in continuous red-shift of the low energy longitudinal mode. This trend reverses as the void growth transitions to the angular phase, and the aspect ratio of the core decreases. Consequently, the longitudinal LSPR mode begins to blue-shift (Fig. 3d). This is reflected in the results, as the spectral position of the longitudinal LSPR mode of $\mathrm{Cu}$ particle reaching a minimum at $\delta \sim 50-60 \%$ during oxidation. 
a
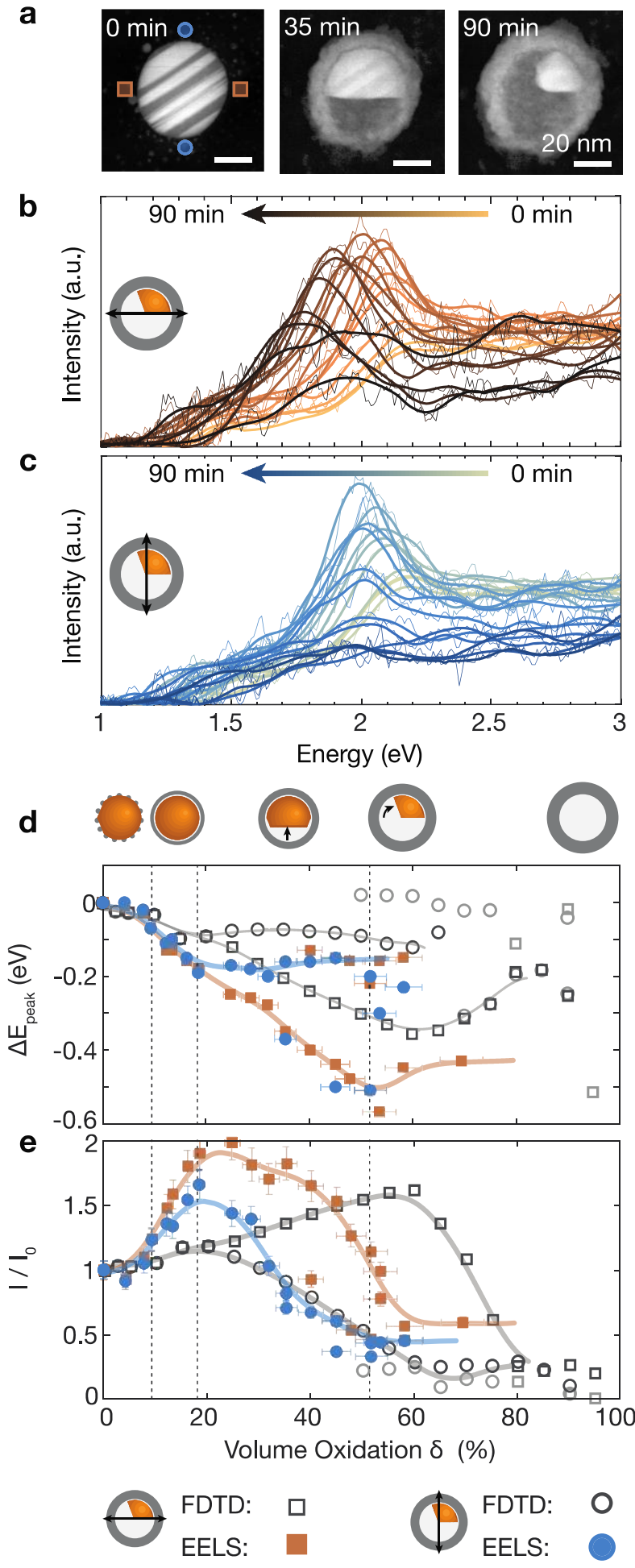

Figure 3. In situ EELS of Cu nanoparticle oxidation at $100^{\circ} \mathrm{C}$. (a) Selected ADF STEM images of a $\mathrm{Cu}$ nanoparticle oxidized at $100{ }^{\circ} \mathrm{C}$ in 3 mbar $\mathrm{O}_{2}$, showing the expansion of the $\mathrm{NK}$ void following first a linear and later angular growth (see Fig. 1 for additional images and Fig. S2.7 for analysis of oxidation at 150 and $200{ }^{\circ} \mathrm{C}$ ). The accumulated $\mathrm{O}_{2}$ exposure time is indicated for each image. All scale bars are $20 \mathrm{~nm}$. (b, c) EELS LSPR signals acquired during oxidation from 
the set of positions indicated by the markers in (a). (d) Peak energy shift, $\Delta E_{\text {peak }}$, and (e) relative peak intensity, $I / I_{0}$, of the LSPR in $(b, c)$ plotted as functions of the particle volume oxidized, $\delta$. The markers (circles and squares) correspond to the positions in (a). Error bars are measured according to the same description in Fig. 2. The experimentally obtained data is plotted together with corresponding FDTD simulations carried out for equivalent excitation polarizations (gray markers). The dashed lines indicate transition between different stages indicated by the schematics: oxide island nucleation, homogeneous oxide shell growth, Kirkendall void formation and transition from linear to angular void expansion.

\section{Unravelling competing oxidation mechanisms}

Starting at $50{ }^{\circ} \mathrm{C}$, the collected evidence suggests that $\mathrm{Cu}_{2} \mathrm{O}$ oxide islands nucleate and grow on the particle surface until they coalesce into a homogeneous oxide shell at $\delta=15 \pm 4 \%$ that corresponds to an effective shell thickness (the total projected oxide area distributed over the whole hemisphere circumference) of around $3 \mathrm{~nm}$ (Fig. 4a-b). This shell grows homogeneously until it reaches $5.5 \pm 0.7 \mathrm{~nm}$ thickness (Fig. $4 \mathbf{c}$ ). This stage of homogeneous shell growth and thickness self-limitation is consistent with the Cabrera-Mott mechanism, for which oxide growth is driven by the Mott potential, $\phi_{\text {Mott }}$. This potential is induced across the oxide layer and decreases in strength upon increasing oxide thickness, thereby leading to self-limiting growth ${ }^{22,45,46}$. Fitting the time evolution of the experimentally determined oxide shell thickness with the Cabrera-Mott model yields good agreement (Fig. 4d). Based on the data obtained from all the particles oxidized at $50{ }^{\circ} \mathrm{C}$, we extract an average Mott potential of $\bar{\phi}_{\text {Mott }}=-3.5 \pm 1 \mathrm{~V}$ and an average energy barrier for extraction and diffusion of cations across the oxide of $\bar{W}=1.1 \pm 0.05 \mathrm{eV}$ (Fig. $4 \mathbf{d d}$ and SI Section S5.1). Considering that the Mott potential can be written as $\phi_{M o t t}=\left(\epsilon+E_{a d s}-\phi_{0}\right) / e$, where $E_{a d s}$ is the $\mathrm{O}^{-}$adsorption energy, $\phi_{0}$ is the metal work function and $\epsilon$ is the electron affinity of $\mathrm{O}$, and using corresponding literature values for $\mathrm{Cu}, \epsilon=1.46 \mathrm{eV}^{47}, E_{a d s}<1 \mathrm{eV}^{48}$ and $4.5 \mathrm{eV}$

$<\phi_{0}<5 \mathrm{eV}^{49-51}$, we estimate a Mott potential in the range $-2 \mathrm{eV}<\phi_{\text {Mott }}<-3 \mathrm{eV}$, which is close to our experimentally obtained value. Similarly, we find the experimentally determined activation barrier, $\bar{W}$, to be in reasonable agreement with the literature ${ }^{22,52}$, which further corroborates a Cabrera-Mott oxidation process at these conditions. As a second important aspect, we note that the observed vacancy gap (Fig. 4b) is formed during the Cabrera-Mott oxidation regime, as $\mathrm{Cu}$ ions get preferentially extracted from the outer perimeter of the metallic particle in order to minimize lattice strain. 

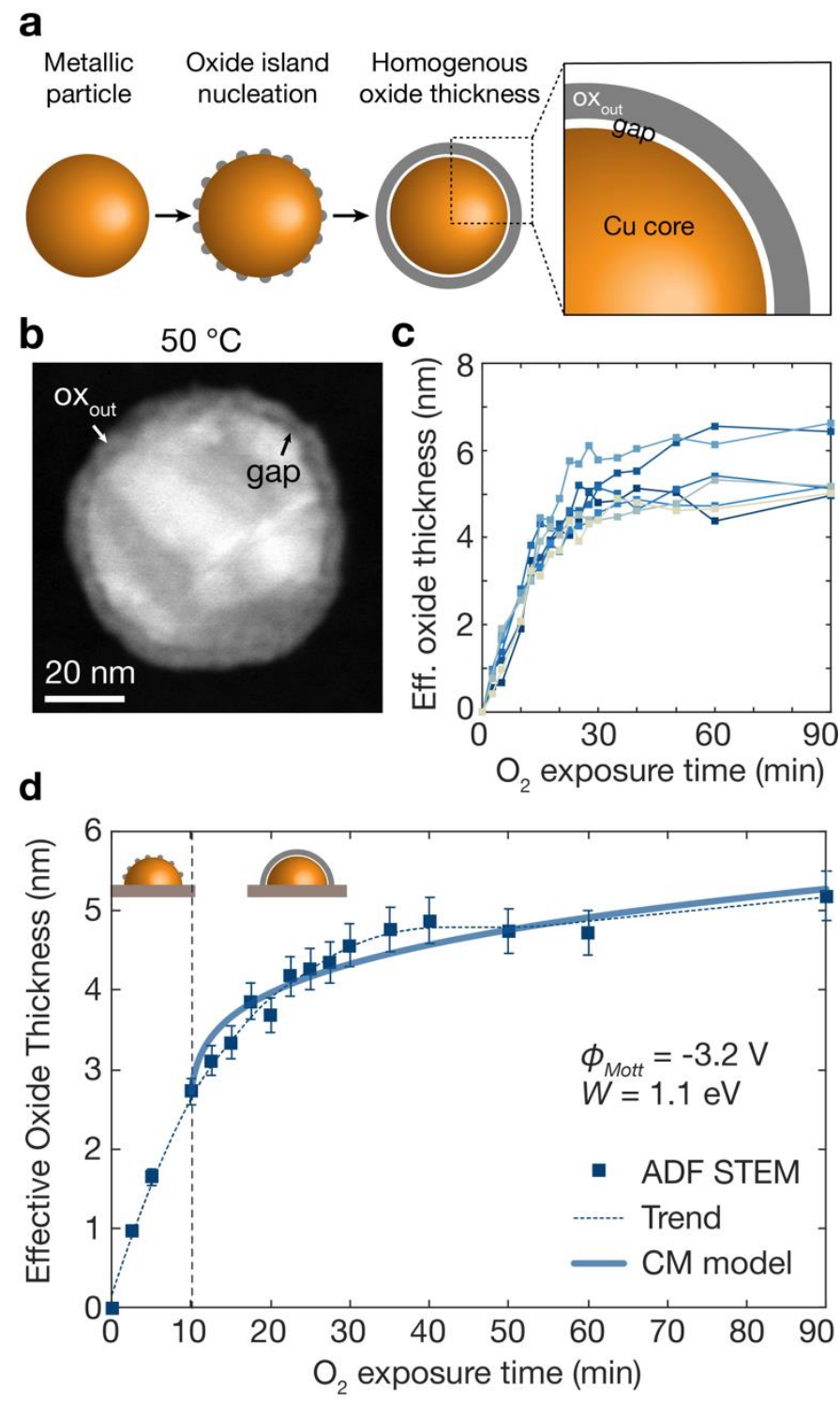

Figure 4: Self-limited oxide growth at $50{ }^{\circ} \mathrm{C}$. (a) A schematic drawing of the oxidation process at $50{ }^{\circ} \mathrm{C}$ including the formation of oxide islands followed by homogenous outer oxide shell separated from the metal core by a vacancy gap layer. (b) ADF STEM image of a particle oxidized at $50^{\circ} \mathrm{C}$ in 3 mbar $\mathrm{O}_{2}$ (same as in Fig. 2a). The outer oxide shell and the vacancy gap are marked. (c) The effective oxide shell thickness measured from ADF STEM images vs. the accumulated $\mathrm{O}_{2}$ exposure time of 7 particles oxidized at $50{ }^{\circ} \mathrm{C}$. (d) Time evolution of the effective oxide shell thickness of the particle in (b), with the error bars showing the uncertainty of the image segmentation process. The dashed line marks the transition from the oxide nucleation phase to a homogeneous oxide shell. The Cabrera-Mott model (solid line) is fitted to the data after the oxide nucleation phase (from $10 \mathrm{~min}$ ) that yields the values for the Mott potential $\phi_{\text {Mott }}=-3.2 \mathrm{eV}$ and the barrier $W=1.1 \mathrm{eV}$. 
Moving to higher oxidation temperatures, as expected, the oxidation rate increases, accompanied by two distinct observations compared to oxidation at $50^{\circ} \mathrm{C}(\mathbf{F i g} .5)$. The first is the aforementioned growth of two oxide shells, separated by a vacancy gap (Fig. 5a-c, cf. Fig 1e), where one oxide shell grows outwards from the surface of the original metallic particle, and a second one grows inwards (Fig. 5b). The location of these two oxide layers relative to the original $\mathrm{Cu}$ particle suggests that the outer oxide layer is the result of $\mathrm{Cu}$ diffusing out, while the inner layer must arise from oxygen diffusing in through the oxide according to the Valensi-Carter mechanism ${ }^{23}$. This process is corroborated by EELS composition mapping (Fig. 5c), where the color map of the relative concentrations reveals the absence of $\mathrm{Cu}$ atoms in the vacancy gap. Furthermore, the analysis confirms that both oxide shells are mainly composed of $\mathrm{Cu}_{2} \mathrm{O}$ (Fig. S2.4b-c), consistent with earlier investigations at similar conditions $\mathrm{s}^{30,31,53}$. Finally, examining the thickness evolution of the inner and outer oxide shells at 100,150 and $200{ }^{\circ} \mathrm{C}$ confirms that the growth rate of both shells increases with temperature, but the outer shell grows faster than the inner shell at all temperatures (Fig. 5e-g).

The second important aspect is that the outward diffusion of $\mathrm{Cu}$, responsible for the outer shell growth, is no longer limited by the Mott-field. Consequently, the oxide grows beyond the selflimiting thickness and the corresponding $\mathrm{Cu}$ extraction leads to a higher concentration of vacancies in the lattice, which introduces tensile strain. The strain magnitude increases with the number of vacancies up to a critical point when it becomes energetically unfavorable to accommodate the strain elastically. This prompts the rapid coalescence of the vacancies, seen as the nucleation of the NK void. From our experiments, we can deduce a critical $\delta=0.22 \pm 0.04$ for NK void nucleation that is independent of particle size (Fig. S2.6). This is consistent with a strain-driven onset of NK void nucleation, where the vacancy density (rather than an absolute number) in the lattice sets the threshold for void formation. Important additional points are the non-uniform strain distribution in the nanoparticles due to the presence of the substrate, and that the inner oxide shell induces further tensile strain at the metal - oxide interface. Both these factors thus lead to the maximum lattice strain being situated at the metal - inner oxide interface, where NK voids thus are expected to nucleate, which is in excellent agreement with our observations (Fig. 1, Fig. S2.2, S2.3). As the final aspect, we highlight that the continued void growth is governed by minimizing the interfacial area between metal and inner oxide shell (Fig. S3), which leads to the experimentally observed transition from a linear to an angular void growth mode at $\delta=60 \pm 10 \%$ (Fig. 3 and Fig. S2.7). In this final oxidation stage, the void continues to expand until the entire particle is consumed and a hollow oxide shell comprised of two layers separated by a vacancy gap is formed. 
a

Metallic Oxide island Outer and inner NK void growth particle nucleation oxide growth

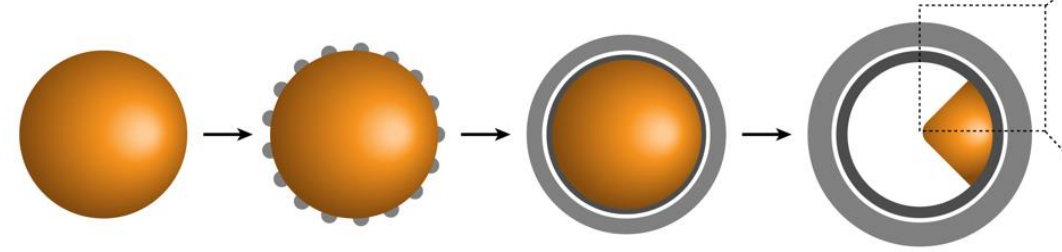

b

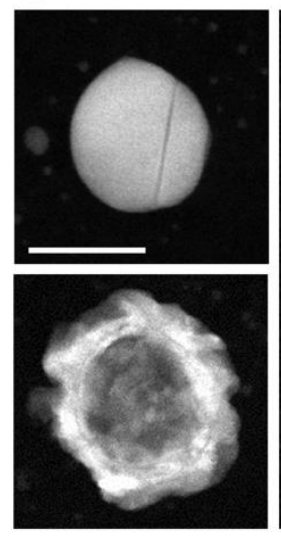

d

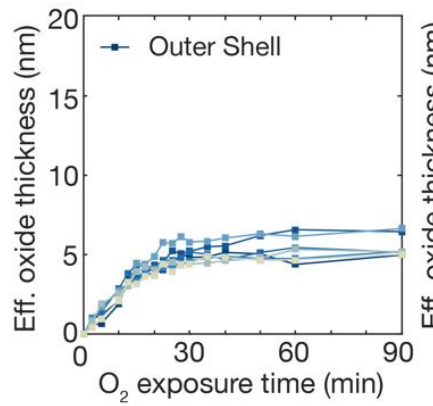

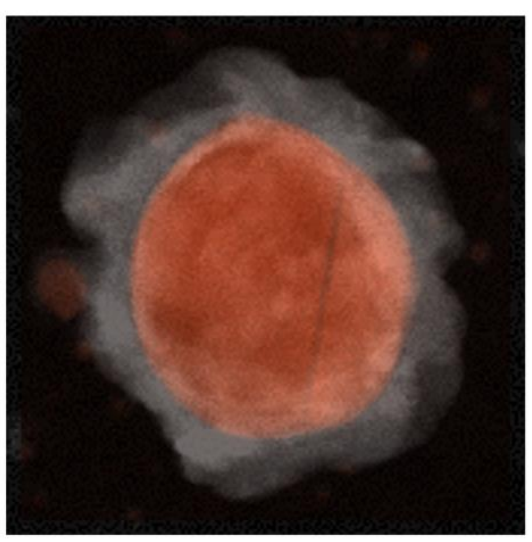

e
C
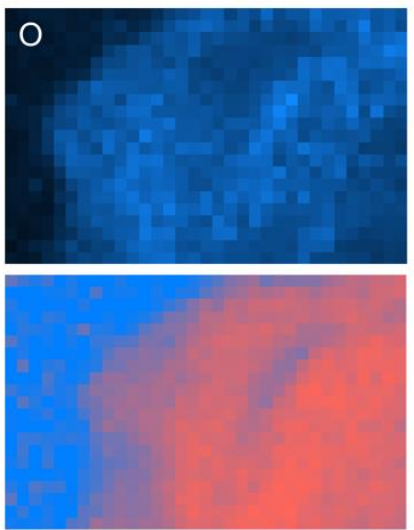

f

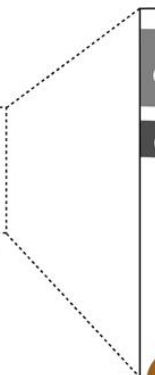

Cu
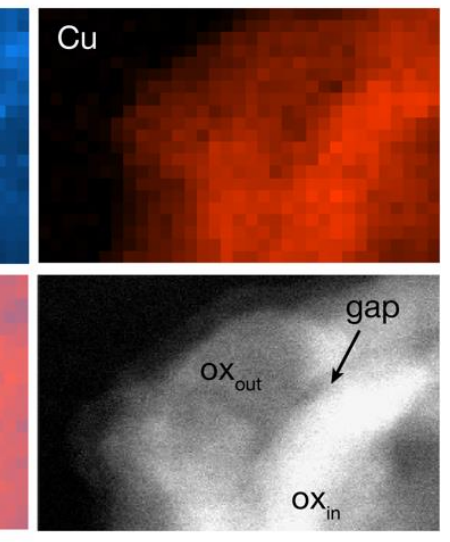

g

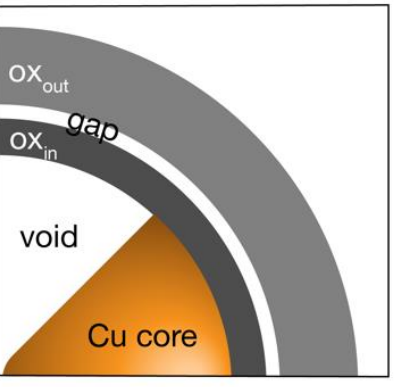

$\mathrm{Cu}$
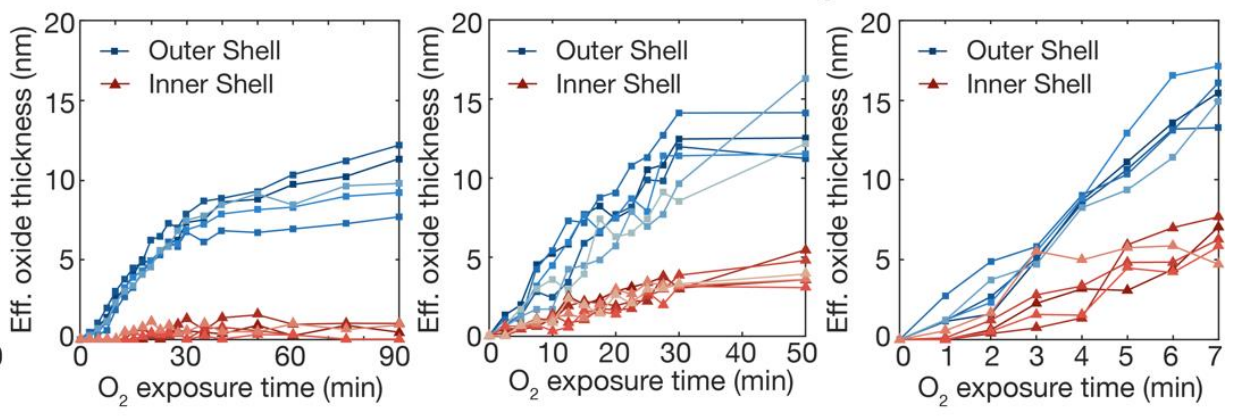

Figure 5. Evidence of competing outer and inner oxide shell growth. (a) Schematics of the stages of oxide growth; the oxide island nucleation, homogenous oxide shell growth - both inner and outer oxide shells, and the formation of the $N K$ void. (b) A particle before (top left) and after (bottom left) oxidation at $200^{\circ} \mathrm{C}$. The scale bar is $50 \mathrm{~nm}$. To the right, the pre and post-oxidation images are overlayed to show the position of the inner and outer oxide shells relative to the perimeter of the pre-oxidation particle (in red). (c) Top row: EELS maps of the $O$ and Cu signals from a section of an oxidized particle. Bottom row: ADF STEM image of the mapped region together with a map of the relative composition of $\mathrm{Cu}$ and $\mathrm{O}$, confirming the lack of $\mathrm{Cu}$ at the vacancy gap between the outer and inner oxide shells. The effective inner (red triangles) and outer (blue squares) oxide shell thicknesses vs. $\mathrm{O}_{2}$ exposure time as extracted from ADF STEM images for a number of single Cu particles oxidized at (d) $50{ }^{\circ} \mathrm{C}$, (e) $100^{\circ} \mathrm{C}$, (f) $150^{\circ} \mathrm{C}$ and (g) $200^{\circ} \mathrm{C}$.

Given our observations, it is apt to quantify the temperature dependence of the growth rates of these oxide shells by means of an Arrhenius analysis. Specifically, we apply the Johnson-Mehl- 
Avrami-Kolmogorov ${ }^{28}$ (JMAK) kinetic model for isothermal nucleation processes to $\delta$ (Fig. 6a), from which we obtain a rate constant, $k$, for each particle (for details see SI Section S5.2). Subsequently, we fit the rate constants to an Arrhenius equation of the form $k=k_{0} \exp \left(-\frac{E_{a}}{k_{B} T}\right)$, where $k_{0}$ is the pre-exponential factor, $E_{a}$ is the apparent activation energy and $k_{B}$ is the Boltzmann constant (Fig. 6b), and extract $E_{a}=0.37 \pm 0.1 \mathrm{eV}$ (for details see SI Section S5.3). This number is in the lower range of previously reported values for $\mathrm{Cu}$ nanoparticles $(0.37-1.5$ $\mathrm{eV})^{30,31,54}$ and $\mathrm{Cu}$ thin films $(0.3-1.0 \mathrm{eV})^{55}$ under similar oxidation conditions. To discriminate between the $E_{a}$ for the growth of the inner and outer oxide shells, the time to reach a specific effective oxide thickness, $\tau$, is compared between the particles oxidized at 100,150 and $200{ }^{\circ} \mathrm{C}$. Here, our analysis yields $E_{\text {out }}=0.30 \pm 0.1 \mathrm{eV}$ for the outer shell to reach $5 \mathrm{~nm}$ and $E_{\text {in }}=0.45 \pm$ $0.1 \mathrm{eV}$ for the inner shell to reach $0.5 \mathrm{~nm}$ (Fig. 6c-d and Fig. S5.8). This is consistent with the lower diffusion coefficient of $\mathrm{O}_{2}{ }^{56}$ compared to that of $\mathrm{Cu}^{57}$ through $\mathrm{Cu}_{2} \mathrm{O}$ at these temperatures, which corroborates our experimental observations. 

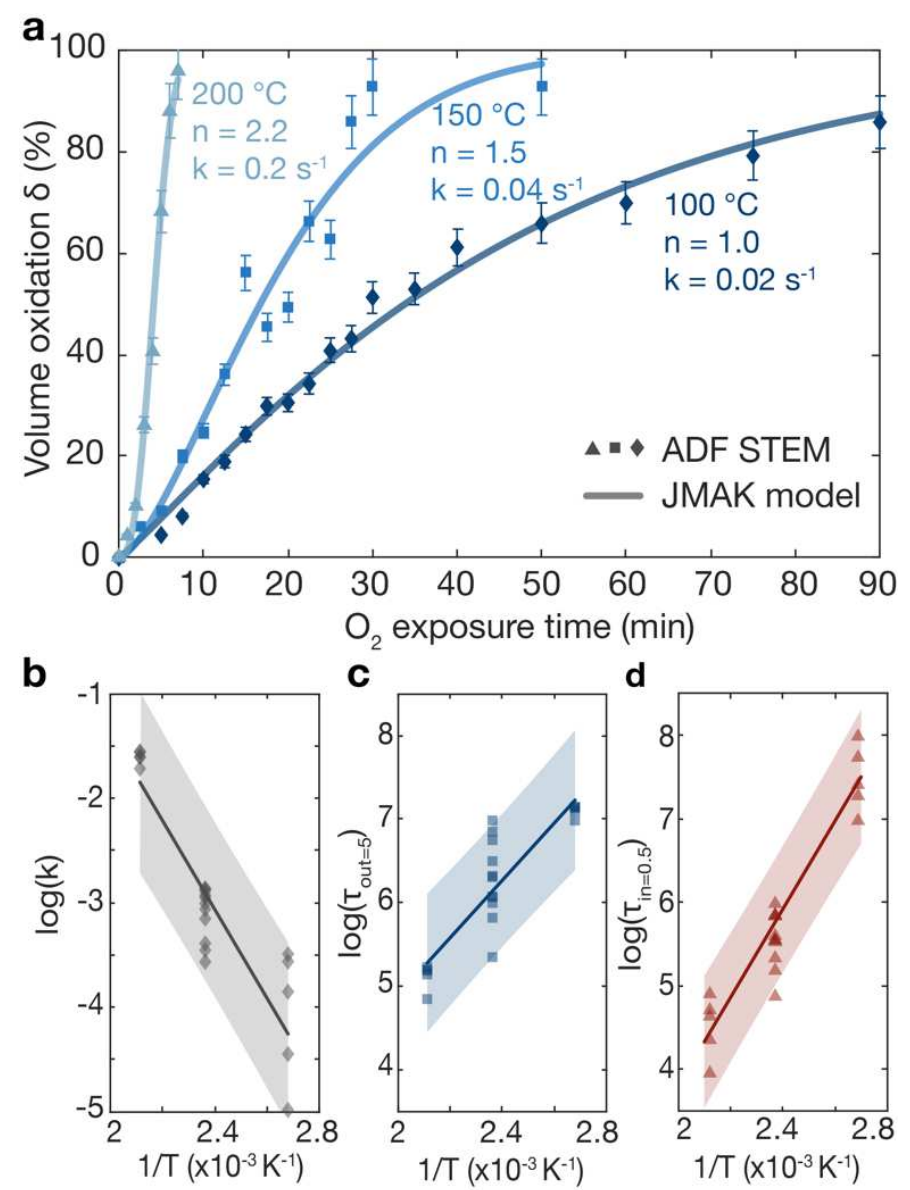

Figure 6. Kinetic analysis of $\mathrm{Cu}$ nanoparticle oxide growth. (a) The volume oxidation fractions vs. $\mathrm{O}_{2}$ exposure times for three $\mathrm{Cu}$ particles oxidized at 100,150 and $200{ }^{\circ} \mathrm{C}$, respectively, obtained from ADF STEM images (error bars show the uncertainty of the image segmentation). The solid lines are fits to the Johnson-Mehl-Avrami-Kolmogorov (JMAK) model with the extracted numbers for parameter $n$ and rate constant $k$ indicated next to each fit. (b) Corresponding Arrhenius plot of $\log (k)$ extracted from JMAK model fits, yielding an overall oxidation $E_{a}=0.37 \pm 0.1 \mathrm{eV} .21$ particles were analyzed and correspond to the individual data points. (c, d) Arrhenius plots for the characteristic times to grow $5 \mathrm{~nm}$ thick outer oxide (c) and $0.5 \mathrm{~nm}$ inner oxide (d), from which the respective apparent activation energies are obtained, $E_{\text {out }}=0.30 \pm 0.1 \mathrm{eV}$ and $E_{\text {in }}=0.45 \pm 0.1$ $\mathrm{eV}$. The shaded areas show the $95 \%$ confidence interval of each fit.

\section{Conclusions}

In summary, we have recorded the oxidation of single $\mathrm{Cu}$ nanoparticles with sub-nanometer spatial resolution at $50-200{ }^{\circ} \mathrm{C}$ using in situ ADF STEM and monitored the evolution of their LSPRs using EELS. Our in situ observations reveal the presence of multiple oxidation mechanisms at play, each with signatures that could be identified in both the morphology of the particles and their plasmonic response. We observe that initially, oxide islands nucleate on the particle surface and subsequently coalesce to form a homogeneous oxide layer separated from the metal core by a nanometer scale vacancy gap at all temperatures. At $50{ }^{\circ} \mathrm{C}$, the Cabrera-Mott mechanism 
dominates, as thermally driven diffusion through the oxide is negligible, which results in a selflimiting oxide shell thickness at this temperature. At temperatures $100-200{ }^{\circ} \mathrm{C}$, concomitant with the outward oxide shell growth, an inner oxide shell grows according to the Valensi-Carter mechanism, arising from the thermally activated inward diffusion of oxygen ions. This inner shell is separated from the outer shell by the vacancy gap. Further on, thermally driven outward diffusion of $\mathrm{Cu}$ ions dominates the outer oxide shell growth and leads to NK void formation at a common oxidation fraction, regardless of particle size. The NK void expansion also follows a common trend across many particles, driven by metal - oxide interface minimization. The signature of these stages driven by their underlying oxidation mechanisms could be traced in the LSPR response of the particles. These insights on competing oxidation mechanisms of metallic nanoparticles are valuable for nanosensors ${ }^{58}$, nanoelectronics ${ }^{59}$ and catalysis ${ }^{60,61}$. More generally, our in situ detection scheme provides opportunities to investigate the intricate details of chemical reactions involving nanoparticles in gaseous environments, and the resulting changes in their optical response. 


\section{Acknowledgements}

SN and CL acknowledge financial support from the Knut and Alice Wallenberg Foundation project 2015.0055. SK acknowledges support from VILLUM FONDEN (research grant 36155). Part of this research has been executed at the Chalmers Nanofabrication Laboratory MC2 and under the umbrella of the Chalmers Excellence Initiative Nano. We thank Prof. V. P. Zhdanov, Dr. T. J. Antosiewicz and Prof. H. Grönbeck for fruitful discussions.

\section{Competing interests}

The authors declare no competing financial interests.

\section{Methods}

Sample fabrication. The $\mathrm{Cu}$ nanoparticles were nanofabricated by means of hole-mask colloidal lithography $(\mathrm{HCL})^{39}$ onto Wildfire chips from DENSsolutions in sparse quasi-random arrays. The steps of the HCL procedure were the following: 1) A thin film Poly(methyl methacrylate) - PMMA A4 - resist in Anisole (MicroChem Corporation) was spin coated at $1500 \mathrm{rpm}$ for $60 \mathrm{~s}$ (acceleration $500 \mathrm{~s}^{-2}$ ), followed by soft-baking on a hotplate at $170{ }^{\circ} \mathrm{C}$ for $5 \mathrm{~min}$. 2) $5 \mathrm{~s} \mathrm{O}_{2}$ plasma treatment (50 $\mathrm{W}, 10 \mathrm{sccm})$ to render the PMMA surface hydrophilic. 3) Drop-coating of poly(diallyldimethylammonium chloride) polyelectrolyte (Sigma Aldrich) diluted in water to 0.2 wt \% and incubated for 40 s. 4) Drop-coating of aqueous sulfonate latex polystyrene (PS) bead suspension with approximate particle sizes of $100 \mathrm{~nm}, 120 \mathrm{~nm}$ and $150 \mathrm{~nm}$, with subsequent $3 \mathrm{~min}$ incubation. 5) Evaporation of $15 \mathrm{~nm} \mathrm{Cr}$ at a rate of $1 \AA / \mathrm{s}$ (Lesker PVD 225), followed by stroking a wet tissue over the substrates $\sim 20$ times to gently remove the PS beads and reveal the holes in the $\mathrm{Cr}$ mask without breaking the TEM membrane. 6) 5 min $\mathrm{O}_{2}$ plasma $(50 \mathrm{~W}, 10 \mathrm{sccm})$ dry etch of the PMMA layer through the holes in the $\mathrm{Cr}$ mask down to the substrate. 7) Evaporation of 20 $\mathrm{nm} \mathrm{Cu}$ at $1 \AA / \mathrm{s}$, followed by lift-off in acetone for approximately $24 \mathrm{~h}$.

ADF STEM imaging and EELS measurements in environmental TEM. The in-situ STEM EELS measurements were carried out in a $80-300 \mathrm{kV}$ FEI Titan environmental TEM equipped with a field emission gun, monochromator and GIF Tridiem spectrometer. The ETEM instrument was operated at $300 \mathrm{kV}$ in STEM mode with an electron probe convergence semi-angle of $10 \mathrm{mrad}$ and with the monochromator excited, giving spatial and energy resolutions of $\sim 0.3 \mathrm{~nm}$ and 0.1 $\mathrm{eV}$, respectively. The fabricated samples were mounted on a DENS Wildfire holder to enable controlled heating of the particles inside the TEM. First, the $\mathrm{Cu}$ nanoparticles were thermally annealed for 60 minutes at $400{ }^{\circ} \mathrm{C}$ in 3 mbar of $\mathrm{H}_{2}$ atmosphere to remove hydrocarbons and to recrystallize into particles containing $1-8$ grains prior to oxidation. Following annealing, the particles were heated to temperatures $50,100,150$ and $200{ }^{\circ} \mathrm{C}$ and oxidized in 3 mbar $\mathrm{O}_{2}$. The particles were exposed to $\mathrm{O}_{2}$ a total time of $90 \mathrm{~min}$ at $50^{\circ} \mathrm{C}$ and $100{ }^{\circ} \mathrm{C}, 50 \mathrm{~min}$ at $150{ }^{\circ} \mathrm{C}$ and 6 
min at $200^{\circ} \mathrm{C}$. ADF STEM images and EELS spectrum images were recorded approximately every $2.5 \mathrm{~min}$ at 50,100 and $150^{\circ} \mathrm{C}$ temperatures and every $1 \mathrm{~min}$ at $200^{\circ} \mathrm{C}$. EELS spectrum images were $10 \times 10$ pixels with a pixel size of $\sim 10 \mathrm{~nm}$. The beam was scanned within each pixel during the acquisitions. The relatively low resolution of the spectrum images was chosen in order to avoid excessive beam induced effects. Each spectrum had a dispersion per channel of $0.01 \mathrm{eV}$ and acquisition time of $10 \mathrm{~ms}$. Prior to each acquisition, the $\mathrm{O}_{2}$ flow was temporarily stopped and the gas was pumped out in order to minimize further oxidation of the nanoparticles when exposed to the electron beam. The $\mathrm{O}_{2}$ flow was resumed again after each acquisition. The data acquired from each particle at different times was combined into 3D image stacks (dimensions $\mathrm{x}, \mathrm{y}$ and time), in the case of the ADF images, and 4D spectrum image series (dimensions $x, y$, energy and time). The spatial drift between different frames in each stack/series was corrected prior to analysis. The EELS spectra were analyzed after normalizing each spectrum with respect to the total area bound by it and the energy-loss axis, followed by removing the contribution from the zero-loss peak by fitting its tail with a power-law model. LSPRs at each time were fitted with Gaussian functions to determine their peak energy and intensity. The ADF STEM images were segmented using the image processing toolbox in MATLAB. The outer particle perimeter was detected automatically by morphological filtering (function imopen, structure element: disk, radius 5 pixels) to reduce noise close to the particle edge and the perimeter was detected using bwboundaries. A circle was fitted to the detected perimeter to calculate the particle radius $R$. The perimeters of the metallic $\mathrm{Cu}$ core, the void, the inner and outer oxide shells were drawn manually (function drawassisted) to measure the area of each phase. From the inner and outer oxide shell area, the effective oxide shell thicknesses were calculated. The oxidation volume fraction after NK void formation was calculated according to the formula for a spherical cap geometry during the linear phase and a spherical sector geometry during the angular phase of void expansion.

Finite-difference time-domain (FDTD) electrodynamic simulations. The Lumerical 2019b software (ANSYS) was used to perform the finite-difference time-domain electrodynamic simulations. The model geometry consisted of a rounded $\mathrm{Cu}$ hemisphere of radius $35 \mathrm{~nm}$ and an edge curvature of $5 \mathrm{~nm}$. This particle was placed on a $25 \mathrm{~nm}$ thin silicon nitride film. Dielectric data for $\mathrm{Cu}$ from reference ${ }^{62}$ and for $\mathrm{Si}_{3} \mathrm{~N}_{4}$ from reference ${ }^{63}$ were used. The inner and outer $\mathrm{Cu}_{2} \mathrm{O}$ oxide shells were simulated using the dielectric data from $\mathrm{Tahir}^{64}$. The correct geometries were calculated at each $\delta$ to achieve the correct oxide volume using the Pilling-Bedworth expansion factor of $1.68^{26}$. For $\delta=0-10 \%$, oxide islands were simulated with height from 2 to $3 \mathrm{~nm}$, and the number of islands calculated based on the total oxide volume. The mesh overlay was $0.25 \mathrm{~nm}$. For $\delta=15-20 \%$, homogenous oxide shell growth was simulated along with the vacancy gap (mesh overlay $0.25 \mathrm{~nm}$ ). Until forming the vacancy shell, the core was shrunk accordingly from R $=35 \mathrm{~nm}$ to $\mathrm{R}=33.2 \mathrm{~nm}$, to account for the conversion of metal to oxide. From $\delta=22 \%$, a NK void was introduced in the model, and up to $60 \%$, the void grew with a linear front (mesh overlay $0.25 \mathrm{~nm}$ ), between 60 and $65 \%$, the void was expanded angularly until full oxidation (mesh 
overlay $0.5 \mathrm{~nm}$ ). Illumination in the simulation was implemented by a total-field scattered-field source incoming from above the particle along the vertical axis, with two perpendicular polarizations that were simulated separately. The absorption cross-section was obtained by integrating the Poynting vector of the absorbed field. The peak(s) were fitted by one or two Lorentzian functions to obtain the peak energies and peak intensities. 


\section{References}

1. Chen, Y. Nanofabrication by electron beam lithography and its applications: A review. Microelectron. Eng. 135, 57-72 (2015).

2. Feng, Y. \& Zhu, J. Copper nanomaterials and assemblies for soft electronics. Science China Materials vol. 62 1679-1708 (2019).

3. Zeng, Y., Bai, K. \& Jin, H. Thermodynamic study on the corrosion mechanism of copper wire bonding. Microelectron. Reliab. 53, 985-1001 (2013).

4. Dubal, D. P., Dhawale, D. S., Salunkhe, R. R., Jamdade, V. S. \& Lokhande, C. D. Fabrication of copper oxide multilayer nanosheets for supercapacitor application. J. Alloys Compd. 492, 26-30 (2010).

5. Böhme, A. et al. Electrochemistry on inverse copper nanoantennas: Active plasmonic devices with extraordinarily large resonance shift. ACS Photonics 6, 1863-1868 (2019).

6. Mkhitaryan, V. et al. Can copper nanostructures sustain high-quality plasmons? Nano Lett. 21, 2444-2452 (2021).

7. Gawande, M. B. et al. $\mathrm{Cu}$ and $\mathrm{Cu}$-based nanoparticles: Synthesis and applications in catalysis. Chem. Rev. 116, 3722-3811 (2016).

8. Muzzio, M. et al. Monodisperse nanoparticles for catalysis and nanomedicine. Nanoscale 11, 18946-18967 (2019).

9. Twigg, M. V. \& Spencer, M. S. Deactivation of copper metal catalysts for methanol decomposition, methanol steam reforming and methanol synthesis. Top. Catal. 22, 191203 (2003).

10. Laudenschleger, D., Ruland, H. \& Muhler, M. Identifying the nature of the active sites in methanol synthesis over $\mathrm{Cu} / \mathrm{ZnO} / \mathrm{A} 12 \mathrm{O} 3$ catalysts. Nat. Commun. 11, 3898 (2020).

11. George H. Chan, Jing Zhao, Erin M. Hicks, Schatz, G. C. \& Duyne, R. P. Van. Plasmonic properties of copper nanoparticles fabricated by nanosphere lithography. Nano Lett. 7, 1947-1952 (2007).

12. Alekseeva, S., Nedrygailov, I. I. \& Langhammer, C. Single particle plasmonics for materials science and single particle catalysis. ACS Photonics 6, 1319-1330 (2019).

13. Chee, S. W., Tan, S. F., Baraissov, Z., Bosman, M. \& Mirsaidov, U. Direct observation of the nanoscale Kirkendall effect during galvanic replacement reactions. Nat. Commun. 8, 1-8 (2017).

14. Anderson, B. D. \& Tracy, J. B. Nanoparticle conversion chemistry: Kirkendall effect, galvanic exchange, and anion exchange. Nanoscale 6, 12195-12216 (2014). 
15. Lee, J. H. Gas sensors using hierarchical and hollow oxide nanostructures: Overview. Sensors and Actuators, B: Chemical vol. 140 319-336 (2009).

16. Guan, W. et al. Gas-sensing performances of metal oxide nanostructures for detecting dissolved gases: A mini review. Front. Chem. 8, 76 (2020).

17. Yin, Y. et al. Formation of hollow nanocrystals through the nanoscale Kirkendall effect. Science 304, 711-714 (2004).

18. Tokozakura, D., Nakamura, R., Nakajima, H., Lee, J.-G. \& Mori, H. Transmission electron microscopy observation of oxide layer growth on $\mathrm{Cu}$ nanoparticles and formation process of hollow oxide particles. J. Mater. Res. 22, 2930-2935 (2007).

19. Railsback, J. G., Johnston-Peck, A. C., Wang, J. \& Tracy, J. B. Size-dependent nanoscale Kirkendall effect during the oxidation of nickel nanoparticles. ACS Nano 4, 1913-1920 (2010).

20. Cabot, A. et al. Vacancy coalescence during oxidation of iron nanoparticles. J. Am. Chem. Soc. 129, 10358-10360 (2007).

21. Sun, Y. et al. Quantitative 3D evolution of colloidal nanoparticle oxidation in solution. Science 356, 303-307 (2017).

22. Cabrera, N. \& Mott, N. F. Theory of the oxidation of metals. Rep. Prog. Phys. 12, (1949).

23. Carter, R. E. Kinetic model for solid-state reactions. J. Chem. Phys. 34, 2010-2015 (1961).

24. Nakamura, R., Tokozakura, D., Nakajima, H., Lee, J.-G. \& Mori, H. Hollow oxide formation by oxidation of Al and Cu nanoparticles. J. Appl. Phys. 101, 074303 (2007).

25. Maack, B. \& Nilius, N. Impact of granularity on the oxidation kinetics of copper. Phys. status solidi 257, 1900778 (2020).

26. Susman, M. D., Vaskevich, A. \& Rubinstein, I. A general kinetic-optical model for solidstate reactions involving the nano Kirkendall effect. The case of copper nanoparticle oxidation. J. Phys. Chem. C 120, 16140-16152 (2016).

27. Yang, J. C., Evan, D. \& Tropia, L. From nucleation to coalescence of $\mathrm{Cu} 2 \mathrm{O}$ islands during in situ oxidation of Cu(001). Appl. Phys. Lett. 81, 241-243 (2002).

28. Fanfoni, M. \& Tomellini, M. The Johnson-Mehl-Avrami-Kolmogorov model: A brief review. Nuovo Cim. della Soc. Ital. di Fis. D - Condens. Matter, At. Mol. Chem. Physics, Biophys. 20, 1171-1182 (1998).

29. Rice, K. P., Paterson, A. S. \& Stoykovich, M. P. Nanoscale Kirkendall effect and oxidation kinetics in copper nanocrystals characterized by real-time, in situ optical 
spectroscopy. Part. Part. Syst. Charact. 32, 373-380 (2015).

30. Susman, M. D., Feldman, Y., Bendikov, T. A., Vaskevich, A. \& Rubinstein, I. Real-time plasmon spectroscopy study of the solid-state oxidation and Kirkendall void formation in copper nanoparticles. Nanoscale 9, 12573-12589 (2017).

31. Albinsson, D., Nilsson, S., Antosiewicz, T. J., Zhdanov, V. P. \& Langhammer, C. Heterodimers for in situ plasmonic spectroscopy: $\mathrm{Cu}$ nanoparticle oxidation kinetics, Kirkendall effect, and compensation in the arrhenius parameters. J. Phys. Chem. C 123, 6284-6293 (2019).

32. LaGrow, A. P., Ward, M. R., Lloyd, D. C., Gai, P. L. \& Boyes, E. D. Visualizing the $\mathrm{Cu} / \mathrm{Cu} 2 \mathrm{O}$ Interface Transition in Nanoparticles with Environmental Scanning Transmission Electron Microscopy. J. Am. Chem. Soc. 139, 179-185 (2017).

33. Nilsson, S., Albinsson, D., Antosiewicz, T. J., Fritzsche, J. \& Langhammer, C. Resolving single $\mathrm{Cu}$ nanoparticle oxidation and Kirkendall void formation with in situ plasmonic nanospectroscopy and electrodynamic simulations. Nanoscale 11, 20725-20733 (2019).

34. Colliex, C., Kociak, M. \& Stéphan, O. Electron energy loss spectroscopy imaging of surface plasmons at the nanometer scale. Ultramicroscopy 162, A1-A24 (2016).

35. Kadkhodazadeh, S. et al. Electron energy loss and one- and two-photon excited SERS probing of "hot" plasmonic silver nanoaggregates. Plasmonics 8, 763-767 (2013).

36. Kadkhodazadeh, S. et al. Scaling of the surface plasmon resonance in gold and silver dimers probed by EELS. J. Phys. Chem. C 118, 5478-5485 (2014).

37. Hansen, T. W. \& Wagner, J. B. Controlled Atmosphere Transmission Electron Microscopy: Principles and Practice. (Springer, Cham, Switzerland, 2016).

38. Baldi, A., Narayan, T. C., Koh, A. L. \& Dionne, J. A. In situ detection of hydrogeninduced phase transitions in individual palladium nanocrystals. Nat. Mater. 13, 1143-1148 (2014).

39. Fredriksson, H. et al. Hole-mask colloidal lithography. Adv. Mater. 19, 4297-4302 (2007).

40. Zhu, Q., Zou, L., Zhou, G., Saidi, W. A. \& Yang, J. C. Early and transient stages of Cu oxidation: Atomistic insights from theoretical simulations and in situ experiments. Surf. Sci. 652, 98-113 (2016).

41. García De Abajo, F. J. Optical excitations in electron microscopy. Rev. Mod. Phys. 82, 209-275 (2010).

42. Tauzin, L. J. et al. Exploring the relationship between plasmon damping and luminescence in lithographically prepared gold nanorods. ACS Photonics 5, 3541-3549 (2018). 
43. Sambles, J. R. Grain-boundary scattering and surface plasmon attenuation in noble metal films. Solid State Commun. 49, 343-345 (1984).

44. Mattsson, A., Panas, I., Siegbahn, P., Wahlgren, U. \& Akeby, H. Model studies of the chemisorption of hydrogen and oxygen on $\mathrm{Cu}(100)$. Phys. Rev. B 36, 7389 (1987).

45. Zhdanov, V. P. \& Kasemo, B. Cabrera-Mott kinetics of oxidation of nm-sized metal particles. Chem. Phys. Lett. 452, 285-288 (2008).

46. Ermoline, A. \& Dreizin, E. L. Equations for the Cabrera-Mott kinetics of oxidation for spherical nanoparticles. Chem. Phys. Lett. 505, 47-50 (2011).

47. Chaibi, W., Peláez, R. J., Blondel, C., Drag, C. \& Delsart, C. Effect of a magnetic field in photodetachment microscopy. Eur. Phys. J. D 2010581 58, 29-37 (2010).

48. Lian, X., Xiao, P., Yang, S. C., Liu, R. \& Henkelman, G. Calculations of oxide formation on low-index Cu surfaces. J. Chem. Phys. 145, 44711 (2016).

49. Anderson, P. A. The work function of copper. Phys. Rev. 76, 388-390 (1949).

50. Singh, B. \& Mehta, B. R. Relationship between nature of metal-oxide contacts and resistive switching properties of copper oxide thin film based devices. Thin Solid Films 569, 35-43 (2014).

51. Greiner, M. T., Chai, L., Helander, M. G., Tang, W. M. \& Lu, Z. H. Transition metal oxide work functions: The influence of cation oxidation state and oxygen vacancies. $A d v$. Funct. Mater. 22, 4557-4568 (2012).

52. Karimi, M., Tomkowski, T., Vidali, G. \& Biham, O. Diffusion of $\mathrm{Cu}$ on $\mathrm{Cu}$ surfaces. Phys. Rev. B 52, 5364 (1995).

53. Mansour, M., Favergeon, L. \& Pijolat, M. Kinetic modeling of low temperature oxidation of copper nanoparticles by O2. Thermochim. Acta 570, 41-50 (2013).

54. Rice, K. P., Walker, E. J., Stoykovich, M. P. \& Saunders, A. E. Solvent-dependent surface plasmon response and oxidation of copper nanocrystals. J. Phys. Chem. C 115, 1793-1799 (2011).

55. Unutulmazsoy, Y. et al. In situ oxidation studies of $\mathrm{Cu}$ thin films: Growth kinetics and oxide phase evolution. J. Appl. Phys. 127, 065101 (2020).

56. Perinet, F., Barbezat, S. \& Monty, C. Transport of oxides: New investigation of oxygen self-diffusion in Cu2O. Le J. Phys. Colloq. 41, C6-315 (1980).

57. Peterson, N. L. \& Wiley, C. L. Diffusion and point defects in Cu2O. J. Phys. Chem. Solids 45, 281-294 (1984).

58. Hübert, T., Boon-Brett, L., Black, G. \& Banach, U. Hydrogen sensors - A review. Sensors 
Actuators, B Chem. 157, 329-352 (2011).

59. Benelmekki, M. \& Erbe, A. Nanostructured thin films-background, preparation and relation to the technological revolution of the 21 st century. in Frontiers of Nanoscience vol. 14 1-34 (Elsevier, 2019).

60. Sambur, J. B. \& Chen, P. Approaches to single-nanoparticle catalysis. Annu. Rev. Phys. Chem. 65, 395-422 (2014).

61. Alayoglu, S. et al. In situ surface and reaction probe studies with model nanoparticle catalysts. ACS Catal. 2, 2250-2258 (2012).

62. McPeak, K. M. et al. Plasmonic films can easily be better: Rules and recipes. ACS Photonics 2, 326-333 (2015).

63. Philipp, H. R. Optical properties of silicon nitride. J. Electrochem. Soc. 120, 295 (1973).

64. Tahir, D. \& Tougaard, S. Electronic and optical properties of $\mathrm{Cu}, \mathrm{CuO}$ and $\mathrm{Cu} 2 \mathrm{O}$ studied by electron spectroscopy. J. Phys. Condens. Matter 24, 175002 (2012). 


\section{Supplementary Files}

This is a list of supplementary files associated with this preprint. Click to download.

- SIfinal.docx 